\title{
Impedance Expressions for Unloaded and Loaded Power Ground Planes
}

\author{
Joe Trinkle and Antonio Cantoni, Fellow, IEEE
}

\begin{abstract}
New, computationally efficient expressions are developed for input and transfer impedance for cylindrical ports on rectangular power ground planes. The expressions show directly the low-frequency characteristics of the planes. This low-frequency behavior is exploited to obtain computationally efficient expressions for impedance parameters of the capacitor-loaded planes that show directly the characteristics of poles caused by the interactions of the loading capacitors and the planes.
\end{abstract}

Index Terms-Decoupling capacitor location, ground plane, impedance, power bus, power plane, printed circuit board (PCB).

\section{INTRODUCTION}

$\mathbf{T}$ HE MANAGEMENT of power supply impedance is an important aspect in the design of high-speed printed circuit boards (PCBs). The magnitude of the impedance at the ports where the components are connected to the supply system determines the amount of supply noise caused by their switching currents. The control of such noise is important for signal integrity, signal referencing, and electromagnetic compatibility. As such, expressions that quantify supply impedance are a useful tool in the design stage of a power distribution system.

In the literature, the power bus is often modeled as a rectangular cavity, and the impedance parameters for the unloaded planes are obtained as an expression involving infinite summations over the cavity modes [1]-[10]. The major contribution of this paper is the derivation of new formulas for the impedance parameters. These new equations offer the following advantages.

1) They use a cylindrical port structure that resembles more closely a via port structure than the rectangular ports used in [1]-[10].

2) The expressions are computationally efficient, involving only one single, rapidly converging, summation. This is achieved without needing to collapse the port into a 1-D structure as is done in [11], and thus, no ad hoc inductance adjustment term is needed. Further, the acceleration technique does not require assumptions regarding the port size as was necessary in [12] to enable the removal of the "sinc" factor. Unlike the traditional summations, the convergence of the new summation is not influenced by the port size and it has a calculable truncation error bound.

Manuscript received October 16, 2006; revised April 26, 2007 and October 4,2007 . This work was supported by the University of Western Australia, Australia.

The authors are with the Western Australian Telecommunications Research Center, School of Electrical and Electronic Engineering, University of Western Australia, Perth, W.A. 6009, Australia (e-mail: trinkl-j@watri.org.au; cantoni@watri.org.au).

Digital Object Identifier 10.1109/TEMC.2008.919036
3) The impedance expressions are conveniently broken into low- and high-frequency characteristics. The lowfrequency characteristics comprise a static capacitance and an equivalent series inductance. The high-frequency component contains the resonance behavior associated with the propagating modes.

4) The low-frequency characteristics can be directly used with the eigenmode decomposition technique outlined in [13] to predict the interactions of many capacitors with the supply planes for frequencies below the first parallel resonance of the planes. These expressions show directly the characteristics of the poles caused by the interactions of the capacitors with the planes. The expressions are also computationally efficient, as they do not require infinite summations to be evaluated, nor do they require the inversion of an impedance matrix for every frequency point as is required in [14].

This paper is organized as follows. In Section II, a new cylindrical port for impedance parameters is defined. In Section III, the impedance parameters are transformed into equivalent expressions that are in terms of line current sources and electric field responses at the center of the ports. In Section IV, this transformation is used in conjunction with the single infinite modal summation of [15]. A convenient separation of the lowand high-frequency components of the impedance parameters is observed. In Section V, the low-frequency components of the impedance parameters are used with the eigen decomposition method [13] to predict the interaction of many loading capacitors with the planes. In Sections VI-VIII, the eigen decomposition method is applied to a typical PCB power bus that is loaded with many capacitors. Observations regarding the location and damping of the poles are made and the computational speed of this method is shown to be far superior to that of doing a matrix inversion for every frequency point.

\section{DEFINITION OF IMPEDANCE PARAMETERS}

Consider the rectangular power ground plane shown in Fig. 1 with $x$-dimension $a, y$-dimension $b$, plane spacing $d$, a dielectric of permittivity $\varepsilon$, and permeability $\mu$. Two ports $p$ and $q$ are shown on the power bus with exaggerated dimensions to allow the port details to be displayed on the same figure. Fig. 1(a) shows the traditional rectangular ports of size $\Delta x \times \Delta y$ and Fig. 1(b) shows the new cylindrical ports of radius $r_{v}$.

A global Cartesian coordinate system is defined with its origin at the bottom left-hand corner of the planes. The location of ports $p$ and $q$, referenced to this coordinate system, are $\left(x_{p}, y_{p}\right)$ and $\left(x_{q}, y_{q}\right)$, respectively. Each port has a local cylindrical coordinate system, as shown in Fig. 1(b). To distinguish between 


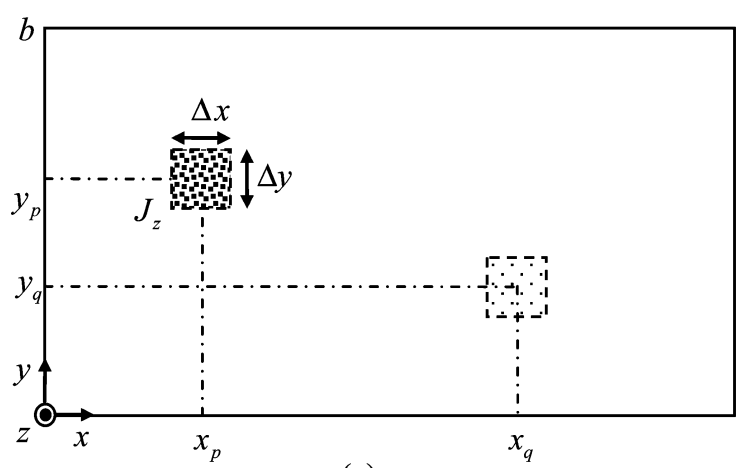

(a)

$a$

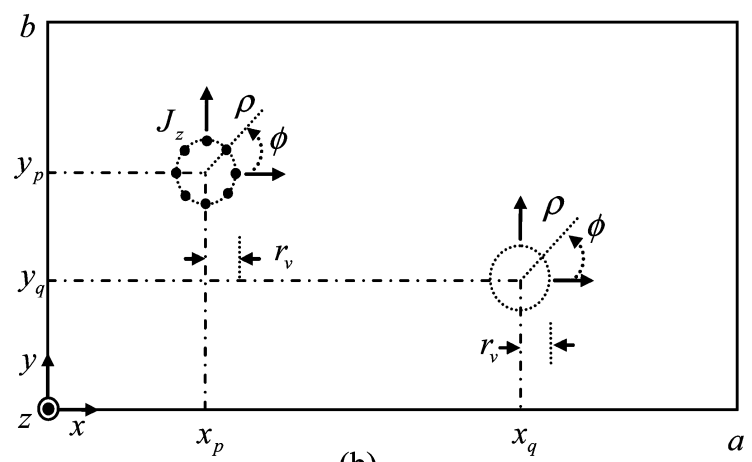

(b)

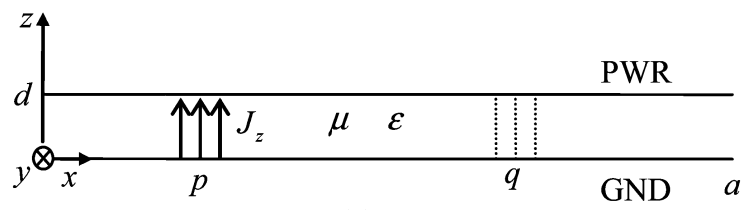

(c)

Fig. 1. Geometry of power bus. (a) Top view of the traditional rectangular ports. (b) Top view of the new, cylindrical port definitions. (c) Side view of the ports.

the different coordinate systems, the first argument of the parameter list of $E_{z}$ is used to indicate the coordinate system to which the parameters are referenced. For example, $E_{z}(0, x, y)$ indicates that $x$ and $y$ are referenced to the global coordinate system, while $E_{z}(p, \rho, \phi)$ indicates that $\rho$ and $\phi$ are referenced to the local coordinate system of port $p$.

Fig. 1(b) shows an injected current distribution of $J_{z}$ between the planes at port $p$ with port $q$ left open. For a source current $I_{p}$ injected symmetrically and cylindrically around the periphery of port $p$ such that

$$
J_{z}(p, \rho, \phi)=\frac{I_{p}}{2 \pi \rho} \delta\left(\rho-r_{v}\right)
$$

with $\delta(\rho)$ being the Dirac delta function, the impedance parameters are defined in terms of the potential difference at the ports, which is assumed to be related to the total electric field $E_{z}$ as

$$
\begin{aligned}
& Z_{q p}=\frac{-d}{2 \pi I_{p}} \int_{0}^{2 \pi} E_{z}\left(q, r_{v}, \phi\right) d \phi \\
& Z_{p p}=\frac{-d}{2 \pi I_{p}} \int_{0}^{2 \pi} E_{z}\left(p, r_{v}, \phi\right) d \phi .
\end{aligned}
$$

These impedance parameters can be used for $N$-port analysis as described in classical microwave theory texts such as [24] and [25]. The following assumptions are made.

A1) The supply planes are perfect electric conductors (PEC) and the open edge walls are perfect magnetic conductors (PMCs).

A2) The metallic vias can be removed, and at the source port, replaced by a uniform impressed cylindrical current $J_{z}$, as shown in Fig. 1(b).

A3) The potential difference (power pane with respect to ground plane) at a port can be obtained by averaging the total electric field $E_{z}$ around a cylinder centered on the port with radius $r_{v}$ and multiplying the result by $-d$.

These assumptions result in a model that has 2-D fields with the electric field being only in the $z$-direction and the magnetic fields only in the $x$ - and $y$ - (or $\rho$ - and $\phi$-) directions. Assumption A1) is true if the planes are good conductors and sufficiently close together so that radiation and fringing effects can be ignored. The effect of finite conductivity can be approximated by allowing a small tangential electric field parallel to the copper planes [20]. Assumptions A2) and A3) require further that the ports be sufficiently small, a sufficient distance apart, and a sufficient distance form the edges of the PCB. If these conditions are not met, coupling effects will disturb the symmetry of the surface currents at the source port, giving rise to errors in the voltages at the ports, and hence, in the impedance values.

\section{INFINITE Plane SOlution}

The electric field between a pair of infinite planes $E_{z}$ at a distance $\rho$ from the center of a cylindrical shell current source of radius $r_{v}$ is [18] ${ }^{1}$

$$
E_{z}\left(p, \rho, \phi, r_{v}\right)=\frac{\omega \mu I_{p}}{-4} \begin{cases}H_{0}^{(2)}\left(k r_{v}\right) J_{0}(p, k \rho) & \rho<r_{v} \\ J_{0}\left(k r_{v}\right) H_{0}^{(2)}(p, k \rho) & \rho \geq r_{v}\end{cases}
$$

where $I_{p}$ is the total injected current, and

$$
k=\omega \sqrt{\mu \varepsilon}(1-j(\tan \delta+r / d))
$$

is the wavenumber [20] with $r=\sqrt{2 /(\omega \mu \sigma)}$ being the skin depth of the electric field in the copper planes and $\tan \delta$ the loss tangent of the dielectric separating them.

If the infinite planes in (4) are truncated to finite rectangular planes of dimensions $a \times b$ with PMC boundaries, the total field produced by a cylindrical source at $p$ can be obtained as an equivalent field using a 2-D grid of image sources on the infinite planes, as shown in Fig. 2. It is shown in [16] that these images are located at $\left(x_{n}\left(x_{p}\right), y_{m}\left(y_{p}\right)\right)$ in relation to the global coordinate system, where

$$
\begin{gathered}
x_{n}=a n-\left\{\begin{array}{c}
x_{p} \\
a-x_{p}
\end{array}\right\},\left\{\begin{array}{l}
n= \pm 0,2,4,6 \ldots \\
n= \pm 1,3,5,7 \ldots
\end{array}\right\} \\
y_{m}=b m-\left\{\begin{array}{c}
y_{p} \\
b-y_{p}
\end{array}\right\},\left\{\begin{array}{l}
m= \pm 0,2,4,6 \ldots \\
m= \pm 1,3,5,7 \ldots
\end{array}\right\} .
\end{gathered}
$$

\footnotetext{
${ }^{1}$ This solution can be obtained by solving the wave equation in cylindrical coordinates subject to the source condition (1) and the radiation boundary condition at infinity. Examples are given in [19] and [22].
} 


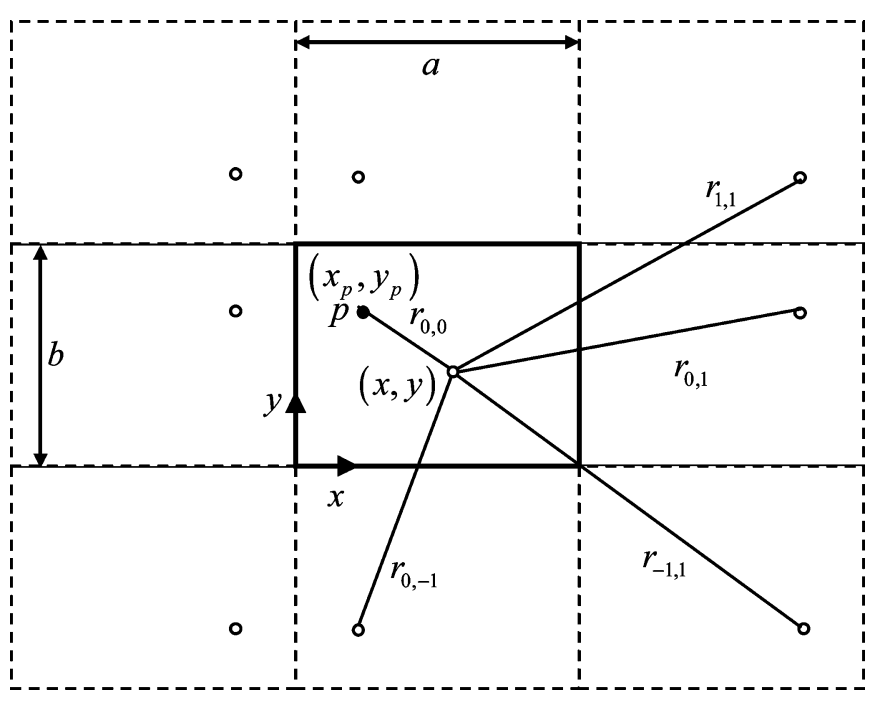

Fig. 2. Location of source and image sources on an infinite pair of planes to account for PMC boundaries. The distances between source points and observation point is denoted as $r_{n, m}$.

The total field between the rectangular planes, referenced to the local coordinate system of a port $q$, is obtained by summing the contributions of the images and the source using (4) as

$$
\begin{aligned}
E_{z}\left(q, \rho, \phi, r_{v}\right)= & \frac{\omega \mu I_{p} J_{0}\left(k r_{v}\right)}{-4} \\
& \times \sum_{\{m, n\}}^{\{I\}} H_{0}^{(2)}\left(k r_{m n}\left(\begin{array}{c}
x_{q}+\rho \cos \phi, \\
y_{q}+\rho \sin \phi
\end{array}\right)\right)
\end{aligned}
$$

where $\{I\}=\{n, m: n \in \mathbf{Z}, m \in \mathbf{Z}\}$ and $\mathbf{Z}$ is the set of all positive and negative integers including zero and $r_{m n}(x, y)$ is the distance between the sources and the observation point $(x, y)$, defined as

$$
r_{m, n}(x, y)=\sqrt{\left(x_{n}-x\right)^{2}+\left(y_{m}-y\right)^{2}}
$$

In a similar way, the electric field referenced to the source port $p$ is

$$
\begin{aligned}
E_{z}\left(p, \rho, \phi, r_{v}\right)= & \frac{\omega \mu I_{p}}{-4} J_{0}\left(k r_{v}\right) \sum_{\{m, n\}}^{\{I\}} H_{0}^{(2)} \\
& \times\left(k r_{m n}\left(x_{p}+\rho \cos \phi, y_{p}+\rho \sin \phi\right)\right)
\end{aligned}
$$

Impedance parameters can now be obtained by substituting the electric field expressions of (7) and (9) into (2) and (3), respectively. The averaging integrations may be done analytically using the addition theorem for Hankel functions [19], [22]. This gives

$$
Z_{q p}=\frac{-d J_{0}\left(k r_{v}\right)^{2}}{I_{p}} E_{z}(q, 0, \phi, 0)
$$

and

$$
Z_{p p}=\frac{\omega \mu d J_{0}\left(k r_{v}\right)^{2}}{4}\left[\frac{-j Y_{0}\left(k r_{v}\right)}{J_{0}\left(k r_{v}\right)}+\lim _{\rho \rightarrow 0}\left(\begin{array}{ll}
\frac{j 2}{\pi} & \ln (k \rho \gamma)- \\
\frac{4 E_{z}(p, \rho, \phi, 0)}{\omega \mu I_{p}}
\end{array}\right)\right]
$$

where $\gamma=0.890522 \ldots$ Note that $E_{z}$ in (10) and (11) is the electric field produced by a line source $\left(r_{v}=0\right)$. This is convenient as the modal summation for $E_{z}$ for a line source excitation does not require any "sinc" factors inside the summation [15]. In the following section, it will be demonstrated that (11) can be used to obtain expressions for impedance parameters that do not have the traditional "sinc" factors, and therefore, do not have their convergence affected by the port size.

\section{Modal Summation}

With reference to the global coordinate system, the electric field at $(x, y)$ for a line source at $\left(x_{p}, y_{p}\right)$ is [15]

$$
\begin{aligned}
& E_{z}^{M}(0, x, y)=\frac{\omega \mu I_{p}}{-j 2 b}
\end{aligned}
$$

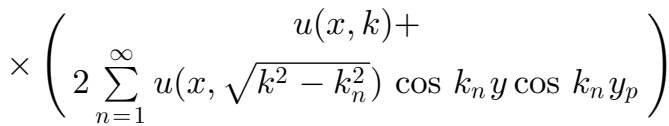

with $k_{n}=n \pi / b$ and

$$
u(x, k)=U\left(x_{p}+x-a, k\right)+U\left(\left|x_{p}-x\right|-a, k\right)
$$

with

$$
U(x, k)=\frac{\cos k x}{k \sin k a} .
$$

The Maclaurin series expansion of $u(x, k)$ for low frequencies (i.e., about $k=0$ ) is ${ }^{2}$

$$
\tilde{u}(x, k)=\frac{2}{a k^{2}}+\frac{a}{3}-\frac{\left(x_{p}+x-a\right)^{2}+\left(\left|x_{p}-x\right|-a\right)^{2}}{2 a} .
$$

For reasons that will become clear in the following sections, it is convenient to rearrange (12) as

$$
\begin{aligned}
& E_{z}^{M}(0, x, y)=\frac{\omega \mu I_{p}}{-j 2 b} \\
& \quad \times\left(\begin{array}{c}
\tilde{u}(x, k)+2 \sum_{n=1}^{\infty} u\left(x, j k_{n}\right) \cos \left(k_{n} y\right) \cos \left(k_{n} y_{p}\right) \\
+u(x, k)-\tilde{u}(x, k)+ \\
2 \sum_{n=1}^{\infty}\left(\begin{array}{c}
u\left(x, \sqrt{k^{2}-k_{n}^{2}}\right) \\
-u\left(x, j k_{n}\right)
\end{array}\right) \cos \left(k_{n} y\right) \cos \left(k_{n} y_{p}\right)
\end{array}\right) .
\end{aligned}
$$

By noting that

$$
E_{z}(q, 0, \phi, 0)=E_{z}^{M}\left(0, x_{q}, y_{q}\right)
$$

\footnotetext{
${ }^{2}$ This expansion can be obtained from a long division of the cosine and sine Maclaurin series and retaining the first two terms.
} 


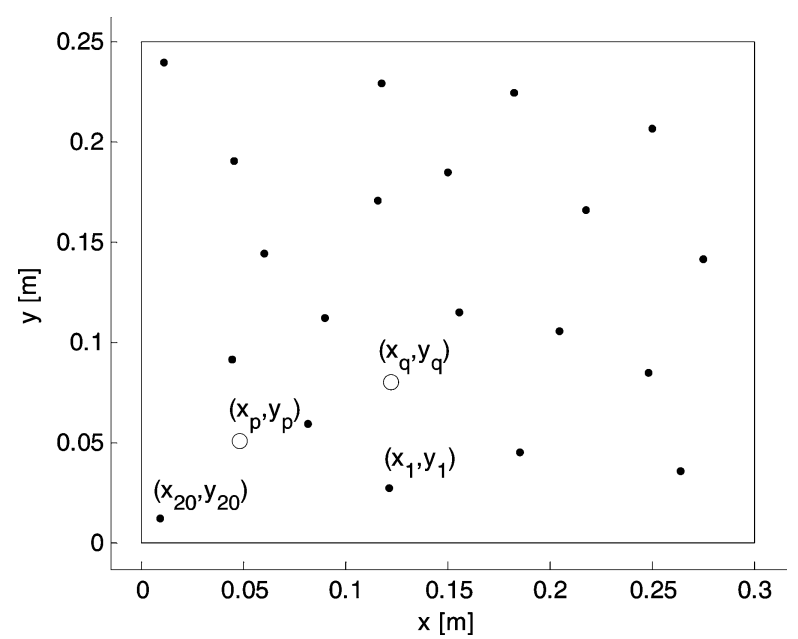

Fig. 3. Power ground plane with $N=20$.

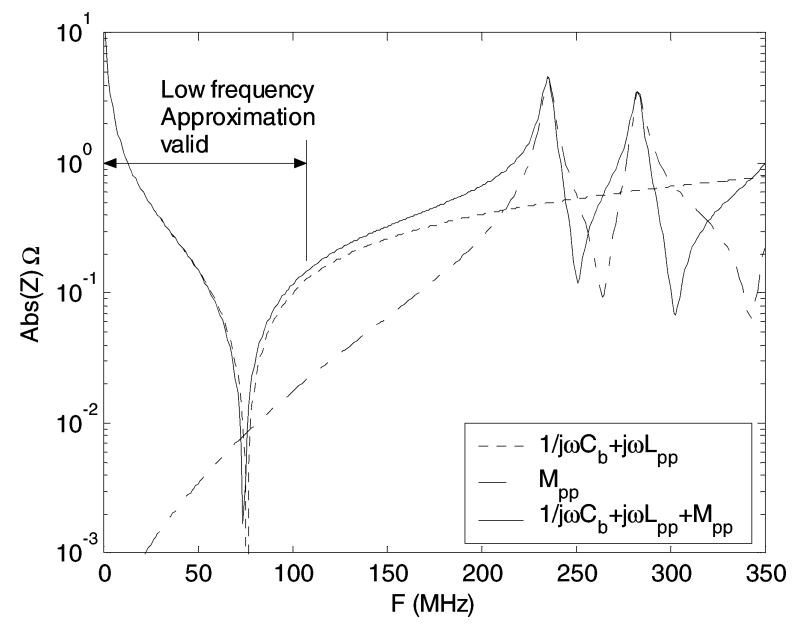

Fig. 4. Different component of the input impedance $Z_{p p}$ expression (20).

and

$$
\lim _{\rho \rightarrow 0} E_{z}(p, \rho, \phi, 0)=\lim _{\rho \rightarrow 0} E_{z}^{M}\left(0, x_{p}+\rho \cos \phi, y_{p}+\rho \sin \phi\right)
$$

it is possible to use (16) in (10) and (11) to give new expressions for the impedance parameters as

$Z_{q p}=J_{0}\left(k r_{v}\right)^{2}\left(\frac{1}{j \omega C_{b}}+j \omega L_{q p}+M_{q p}(\omega)\right)$

$Z_{p p}=J_{0}\left(k r_{v}\right)^{2}\left(\frac{1}{j \omega C_{b}}+j \omega L_{p p}\left(r_{v}\right)+M_{p p}\left(\omega, r_{v}\right)\right)$.

In Fig. 4, the input impedance $Z_{p p}$ according to (20) is shown for the PCB of Fig. 3 with parameters as given in Table I. The $N=20$ capacitor ports are defined for later connection of decoupling capacitors. For the results in Fig. 4, these ports are left open circuit and can be ignored. The factor $J_{0}\left(k r_{v}\right)^{2} \approx 0.999996 \approx 1$ at the highest frequency of $350 \mathrm{MHz}$ and so can be set to 1 with negligible error. Fig. 4 shows that, for frequencies below $100 \mathrm{MHz}$, the first two terms, namely the static capacitance and the series inductance are a good approximation for the complete expression. The third term $M_{p p}$ contributes an insignificant amount for frequencies below $100 \mathrm{MHz}$, but has a significant influence at higher frequencies and contributes the resonance behavior associated with the propagating modes.

Equations (19) and (20) give the impedance parameters conveniently broken up into the static capacitance, the series inductance, and the resonance effects caused by the propagating modes. The following sections describe the different parts of the expressions.

\section{A. Static Capacitance Term}

The first terms in the brackets of (19) and (20) represent the static capacitance of the parallel planes, defined as

$$
C_{b}=\frac{a b \varepsilon}{d} \text {. }
$$

This part of the impedance is dominates at very low frequencies. It is obtained from the first term of $\tilde{u}\left(x, x_{p}, k\right)$.

\section{B. Series Inductance Term}

The second terms in the brackets of (19) and (20) correspond to equivalent series inductances

$$
\begin{aligned}
L_{q p} & =L_{1}\left(x_{q}\right)+L_{2}\left(x_{q}, y_{q}\right) \\
L_{p p}\left(r_{v}\right) & =L_{1}\left(x_{p}\right)+\lim _{\rho \rightarrow 0}\left[\begin{array}{c}
L_{2}\left(x_{p}+\rho \cos \phi, y_{p}+\rho \sin \phi\right) \\
+\frac{\mu d}{2 \pi} \ln \left(\frac{\rho}{r_{v}}\right)
\end{array}\right]
\end{aligned}
$$

where $L_{1}$ represents an inductance term that is obtained from $\tilde{u}\left(x, x_{p}, k\right)$ as

$L_{1}(x)=\mu d\left(\frac{\left(x_{p}+x-a\right)^{2}+\left(\left|x_{p}-x\right|-a\right)^{2}}{4 a b}-\frac{a}{6 b}\right)$.

The inductance $L_{1}$ corresponds to the series inductance seen looking into the parallel combination of two transmission lines, one of length $x_{p}$ and the other of length $a-x_{p}$. This inductance term is not a function of the port radius. The term $L_{2}(x, y)$ is obtained from the second term in the brackets of (16) as

$$
L_{2}(x, y)=\frac{\mu d}{-b} \sum_{n=1}^{\infty} u\left(x, j k_{n}\right) \cos \left(k_{n} y\right) \cos \left(k_{n} y_{p}\right) .
$$

These terms involve summations of evanescent modes and correspond to the extra inductance due to the fact that the port is cylindrical and does not only excite propagating modes but also evanescent modes.

It is shown in the Appendix that these summations yield a closed-form solution for inductance (with error $<0.2 \%$ for $a>b)$ as

$$
\begin{aligned}
L_{q p} & \approx L_{1}\left(x_{q}\right)-\frac{\mu d}{2 \pi} \ln \left(F_{q p}\left(x_{p}, x_{q}, y_{p}, y_{q}\right)\right) \\
L_{p p}\left(r_{v}\right) & \approx L_{1}\left(x_{p}\right)-\frac{\mu d}{2 \pi} \ln \left(F_{p p}\left(x_{p}, y_{p}, r_{v}\right)\right)
\end{aligned}
$$


where the functions $F_{q p}$ and $F_{p p}$ are defined in Appendix A. It is clear from (25) that the transinductance $L_{q p}$ is not a function of the via radius but the input inductance $L_{p p}$ is a function of the via radius.

\section{Propagating Term}

The final terms in the bracketed part of the impedance expressions in (19) and (20) represent the impedance due to the propagating modes

$$
\begin{aligned}
& M_{q p}=M_{1}\left(\omega, x_{q}\right)+M_{2}\left(\omega, x_{q}, y_{q}\right) \\
& M_{p p}=M_{1}\left(\omega, x_{p}\right)+M_{2}\left(\omega, x_{p}, y_{p}\right)+M_{3}\left(\omega, r_{v}\right) .
\end{aligned}
$$

The first term $M_{1}$ represents the propagating modes associated with a transmission line in the $x$-dimension and the final terms $M_{2}$ and $M_{3}$ represent the propagating modes that have additional $y$ or $r_{v}$ dependence. They are obtained from the third and fourth terms in the brackets of (16) as

$$
\begin{aligned}
M_{1}(\omega, x)= & \frac{j \omega \mu d}{-2 b}(u(x, k)-\tilde{u}(x, k)) \\
M_{2}(\omega, x, y)= & \frac{j \omega \mu d}{-b} \sum_{n=1}^{\infty}\left(\begin{array}{c}
u\left(x, \sqrt{k^{2}-k_{n}^{2}}\right) \\
-u\left(x, j k_{n}\right)
\end{array}\right) \\
& \times \cos \left(k_{n} y\right) \cos \left(k_{n} y_{p}\right) \\
M_{3}\left(\omega, r_{v}\right)= & \frac{j \omega \mu d}{4}\left(\frac{2}{\pi} \ln \left(k r_{v} \gamma\right)-\frac{Y_{0}\left(k r_{v}\right)}{J_{0}\left(k r_{v}\right)}\right) .
\end{aligned}
$$

The $M_{2}$ term involves an infinite summation. The summation can be truncated after few terms as it converges rapidly. The convergence of the summation is not affected by the port size, as is the case for the double summation formulas [1]-[10]. It is shown in the Appendix B that its rate of convergence for $M$ terms is $O\left[1 / M^{2}\right]$ or better and that the truncation error is bounded approximately by (51).

In Fig. 5 the input impedance $Z_{p p}$ according to (19) is compared with the input impedance for the double summation technique [see (52)] for the geometry of Fig. 3 and Table I without capacitors and with a port size of $r_{v}=1 \mathrm{~mm}$.

For the double summation, a small square port as in Fig. 1(a) with sides $\Delta x=\Delta y=\pi r_{v} / 2$ so as to have the same circumference as the cylindrical port is used. For the evaluation of (19), only ten terms are used for the summation. For the double sum, at least $80 \times 80=1600$ terms are required to give the same result.

The PCB should be oriented with the longest dimension in the $x$-axis (i.e., $a \geq b$ ) so that the $0.2 \%$ error bound for the inductance (42) and the truncation error bound (51) as shown in the Appendix are valid.

Finally, it is interesting to note that, with the exception of the $\left(J_{0}\left(k r_{v}\right)\right)^{2}$ term, the transfer impedance is not a function of the port size $r_{v}$.

\section{LOADED PLANES}

The addition of decoupling capacitors to the power ground planes can be modeled by adding impedance loads to the ap-

\begin{tabular}{|c|c|c|c|c|c|c|c|}
\hline Paran & Val. & Pt. & $x(m)$ & $y(m)$ & Pt. & $x(m)$ & $y(m)$ \\
\hline$a$ & $0.3 m$ & 1 & 0.0899 & 0.1123 & 11 & 0.2496 & 0.2061 \\
\hline$b$ & $0.25 \mathrm{~m}$ & 2 & 0.0811 & 0.0596 & 12 & 0.2478 & 0.0851 \\
\hline$d$ & $0.25 \mathrm{~mm}$ & 3 & 0.1215 & 0.0272 & 13 & 0.2636 & 0.0360 \\
\hline \multirow[t]{2}{*}{$r_{\nu}$} & $0.25 \mathrm{~mm}$ & 4 & 0.0443 & 0.0912 & 14 & 0.2750 & 0.1412 \\
\hline & & 5 & 0.0452 & 0.1904 & 15 & 0.0601 & 0.1447 \\
\hline \multirow{2}{*}{\multicolumn{2}{|c|}{ Material Param. }} & 6 & 0.1496 & 0.1851 & 16 & 0.1180 & 0.2289 \\
\hline & & 7 & 0.2048 & 0.1061 & 17 & 0.2171 & 0.1658 \\
\hline $\tan \delta$ & 0.02 & 8 & 0.1855 & 0.0456 & 18 & 0.1820 & 0.2246 \\
\hline$\varepsilon_{r}$ & 4.42 & 9 & 0.1557 & 0.1149 & 19 & 0.0110 & 0.2395 \\
\hline \multirow[t]{2}{*}{$\mu$} & $\mu_{0}$ & 10 & 0.1154 & 0.1711 & 20 & 0.0092 & 0.0123 \\
\hline & & $p$ & 0.0487 & 0.0509 & $q$ & 0.1224 & 0.0807 \\
\hline
\end{tabular}

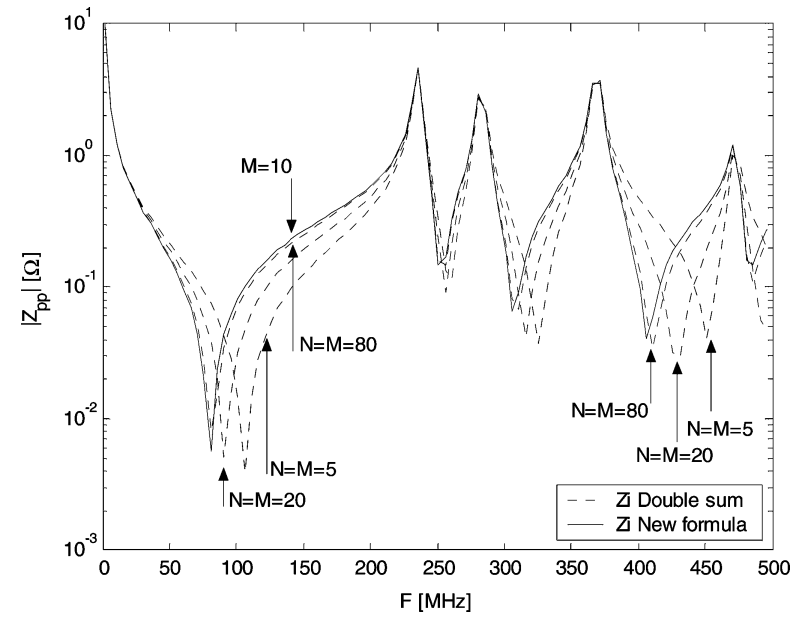

Fig. 5. Comparison of new input impedance formula (20) with traditional double summation formula (52).

TABLE I

PCB PARAMETERS

propriate ports. Assume that $N$ capacitors are added to ports 1 to $N$ defined by their locations $\left(x_{1}, y_{1}\right),\left(x_{2}, y_{2}\right)$ to $\left(x_{N}, y_{N}\right)$. Assume further that all capacitors are of the same value and that their loading impedance at the ports can be modeled as a lumped inductor-capacitor-resistance (LCR) series circuit as

$$
Z_{d}(\omega)=\frac{1}{j \omega C_{d}}+j \omega L_{d}+R_{d}
$$

where $C_{d}$ is the capacitance of each of the added capacitors, $L_{d}$ is the combination of the capacitors equivalent series inductance (ESL) and mounting inductance, and $R_{d}$ is the capacitors equivalent series resistance (ESR).

Using $N$-Port circuit theory as in [13] and [14] the loaded input and transfer impedance for ports $p$ and $q$ (in terms of their unloaded impedances) are

$$
\left[\begin{array}{cc}
Z_{p p} & Z_{p q} \\
Z_{q p} & Z_{q q}
\end{array}\right]=\left[\begin{array}{cc}
Z_{p p} & Z_{p q} \\
Z_{q p} & Z_{q q}
\end{array}\right]-\left[\begin{array}{cc}
Z_{p p}^{\Delta} & Z_{p q}^{\Delta} \\
Z_{q p}^{\Delta} & Z_{q q}^{\Delta}
\end{array}\right]
$$

where the superscript " “ is used to denote the loaded impedance parameters. The second matrix on the right-hand 
side of (31) requires the inversion of an $N \times N$ matrix

$$
\left[\begin{array}{cc}
Z_{p p}^{\Delta} & Z_{p q}^{\Delta} \\
Z_{q p}^{\Delta} & Z_{q q}^{\Delta}
\end{array}\right]=\left[Z_{q p N}\right]^{T}\left[\left[Z_{N N}\right]+Z_{d}[I]\right]^{-1}\left[Z_{q p N}\right]
$$

where $\left[Z_{N N}\right]$ is the $N \times N$ impedance matrix corresponding to the capacitor ports, $\left[Z_{q p N}\right]$ is a $2 \times N$ impedance matrix and corresponds to the transfer impedances between ports $q, p$ and the $N$ capacitor ports. For low-frequency analysis, these matrices can be approximated as

$$
\begin{aligned}
& {\left[Z_{q p N}\right] \approx \frac{1}{j \omega C_{b}}\left[\begin{array}{l}
\hat{o}_{N} \\
\hat{o}_{N}
\end{array}\right]+j \omega\left[\begin{array}{l}
\hat{l}_{p} \\
\hat{l}_{q}
\end{array}\right]} \\
& {\left[Z_{N N}\right] \approx \frac{1}{j \omega C_{b}} \hat{o}_{N}^{T} \cdot \hat{o}_{N}+j \omega\left[L_{N N}\right]}
\end{aligned}
$$

where $\hat{l}_{p}$ and $\hat{l}_{q}$ are $1 \times N$ vectors containing the series inductance associated with transfer impedances from ports $p$ and $q$ to the $N$ capacitor ports, respectively. The $N \times N$ matrix $\left[L_{N N}\right]$ contains the inductances associated with the $N$ capacitor ports. The vector $\hat{o}_{N}$ is of length $N$ and contains all $1 \mathrm{~s}$. All these inductance parameters can be obtained using (22). If the eigen vectors and eigen values of $\left[L_{N N}\right]$ are $\hat{v}^{i}$ and $\lambda_{i}$, respectively, then, with the following definitions:

$$
\begin{aligned}
\alpha_{i} & =\hat{o}_{N} \cdot \hat{v}^{i} \quad \beta_{i}^{p}=\hat{l}_{p} \cdot \hat{v}^{i} \quad \beta_{i}^{q}=\hat{l}_{q} \cdot \hat{v}^{i} \\
y_{i}(\omega) & =\frac{1}{Z_{d}(\omega)+j \omega \lambda_{i}} \\
\alpha(\omega) & =\sum_{i=1}^{N} y_{i}(\omega) \alpha_{i}^{2} \\
\beta^{p}(\omega) & =\sum_{i=1}^{N} y_{i}(\omega) \alpha_{i} \beta_{i}^{p} \\
\beta^{q}(\omega) & =\sum_{i=1}^{N} y_{i}(\omega) \alpha_{i} \beta_{i}^{q} \\
f\left(\omega, \alpha, \beta^{p}, \beta^{q}\right) & =\left(\frac{\alpha}{j \omega C_{b}}+j \omega \beta^{p}\right)\left(\frac{\alpha}{j \omega C_{b}}+j \omega \beta^{q}\right)
\end{aligned}
$$

the $Z_{q p}^{\Delta}$, element in (32), is [13]

$$
\begin{aligned}
Z_{q p}^{\Delta}(\omega) \approx & \sum_{i=1}^{N} y_{i}(\omega) f\left(\omega, \alpha_{i}, \beta_{i}^{p}, \beta_{i}^{q}\right) \\
& -\frac{f\left(\omega, \alpha(\omega), \beta^{p}(\omega), \beta^{q}(\omega)\right)}{j \omega C_{b}+\alpha(\omega)} .
\end{aligned}
$$

The remaining parameters $Z_{p p}^{\Delta}, Z_{q q}^{\Delta}$, and $Z_{p q}^{\Delta}$ can be obtained from (40) by interchanging $p$ and $q$.

\section{CASE STUdY}

The use of (31) with (40) for analyzing the loading effect of multiple capacitors can be demonstrated by considering, as an example, the power ground planes shown in Fig. 3 with the parameters of Table I. Twenty capacitors $(N=20)$ are connected to the ports indicated. Ports $p$ and $q$ are located as shown and

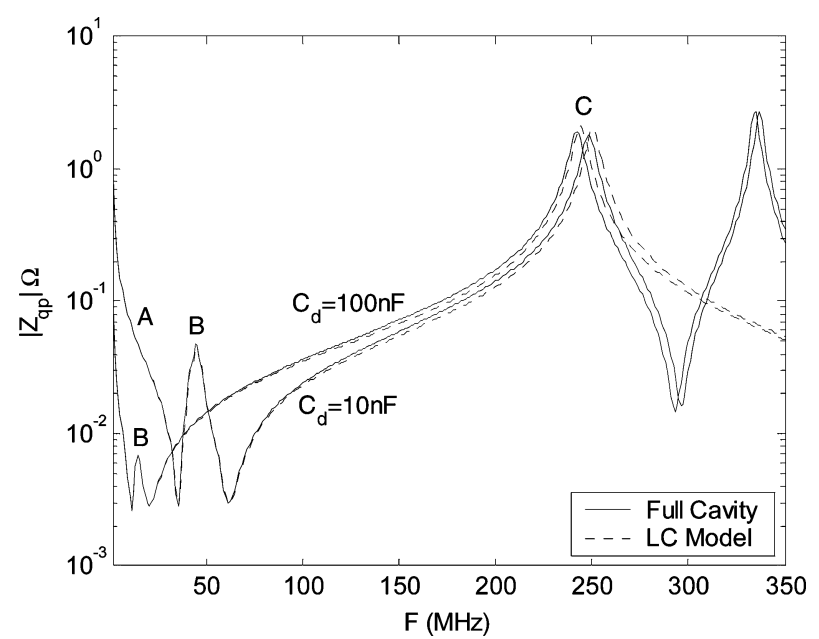

Fig. 6. Transfer impedance between ports $p$ and $q$ for $C_{d}=10$ and $100 \mathrm{nF}$ with $\varepsilon_{r}=4.24$ such that $C_{b}=11.7 \mathrm{nF}$.

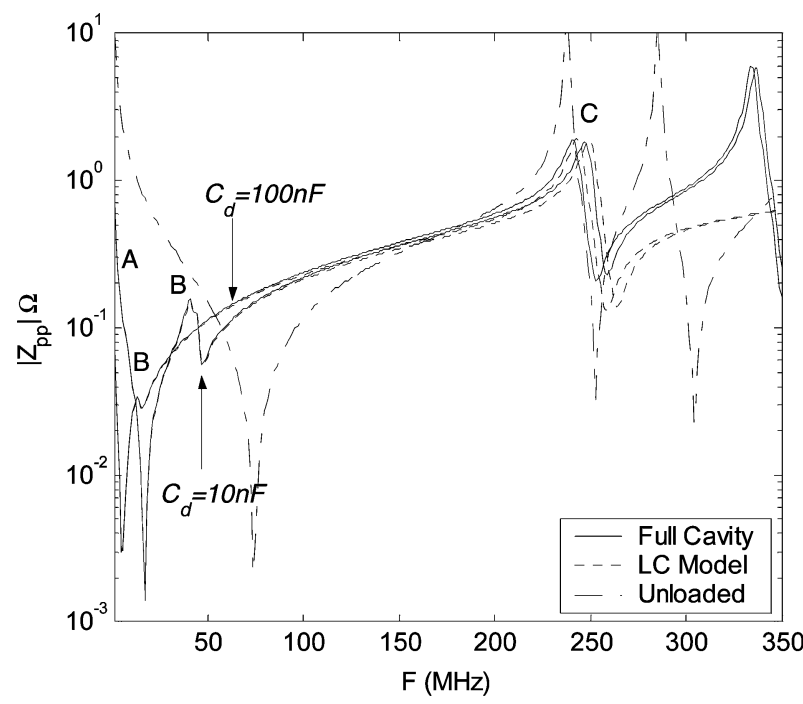

Fig. 7. Input impedance between ports $p$ and $q$ for $C_{d}=10$ and $100 \mathrm{nF}$ with $\varepsilon_{r}=4.24$ such that $C_{b}=11.7 \mathrm{nF}$.

are used to observe the behavior of the loaded planes. The decoupling capacitors are all of equal value $C_{d}$, and have parasitic values $L_{d}=0.5 \mathrm{nH}$ and $R_{d}=30 \mathrm{~m} \Omega$.

The loaded transfer impedances are first calculated using the full cavity solution of (19) and (20) in (32) and (31) to give impedance values that are correct for a wide range of frequencies. This method is referred to as the "full cavity" method. The loaded low-frequency parameters are then calculated according to (31) but by using the low-frequency approximations (40) for $Z_{q p}^{\Delta}, Z_{p p}^{\Delta}, Z_{q q}^{\Delta}$, and $Z_{p q}^{\Delta}$. This method is referred to the "LC method." The calculations are done up to $350 \mathrm{MHz}$ so as to include the first two cavity resonances of the structure.

The loaded parameters $\widehat{Z}_{q p}$ and $\bar{Z}_{p p}$ are shown in Figs. 6 and 7 respectively for $C_{d}=10$ and $100 \mathrm{nF}$. 


\section{DisCUSSION OF RESUlTS}

The results presented in Figs. 6 and 7 will be discussed by considering three frequency regions indicated as $A, B$, and $C$.

\section{A. Low-Frequency Region}

In the low-frequency region, all the impedances are governed by the combined effect of the capacitance of the planes $C_{b}$ and the total added decoupling $N \times C_{d}$. In this region, the agreement between the full cavity and LC methods is very good.

\section{B. Capacitor Interaction Region}

The next frequency region contains the interaction of the decoupling capacitors $C_{d}$ with each other by means of the planes. These interactions are a consequence of the poles of $y_{i}(\omega)$ in (35). The poles occur at $f_{i}=1 /\left(2 \pi \sqrt{C_{d}\left(L_{d}+\lambda_{i}\right)}\right)$ where $i=1,2,3, \ldots, N$. Thus, there is one pole for every capacitor. The spread of the poles with frequency is determined by the spread of the eigen values $\lambda_{i}$, which, in turn, depends on the location of the ports. The larger the value of $C_{d}$, the lower these poles occur in the frequency spectrum. This is demonstrated in Figs. 6 and 7.

The damping of the poles ${ }^{3} \zeta_{i}=\left(R_{d} / 2\right) \sqrt{C_{d} /\left(L_{d}+\lambda_{i}\right)}$ is proportional to $\sqrt{\mathrm{C}_{d}}$ for constant $L_{d}+\lambda$ and $R_{d}$. The influence of varying $C_{d}$ on the damping of these poles is seen in Figs. 6 . and 7 . The individual poles are not visible as they are closely spaced and $R_{d}=30 \mathrm{~m} \Omega$ provides too much damping for them to appear distinct. For this region, there is a very good agreement between the full cavity and LC methods.

\section{Capacitor-Plane Interaction Region}

The next frequency region consists of a single, well-defined pole that is easy to distinguish from the capacitor interaction region. This pole corresponds to the frequency for which the denominator of the second term in (40) is minimized. For $\omega \gg$ $\omega_{i}$ 's, the denominator can be approximated as

$$
\begin{aligned}
j \omega C_{b}+\sum_{i=1}^{N} y_{i}(\omega) \alpha_{i}^{2} & \approx j \omega C_{b}+\frac{1}{j \omega} \sum_{i=1}^{N} \frac{\alpha_{i}^{2}}{L_{d}+\lambda_{i}} \\
& =j \omega C_{b}+\frac{1}{j \omega L_{D}}
\end{aligned}
$$

where $L_{D}$ is a combination of all the $L_{d}+\lambda_{i}$ terms. This $L_{D}$ interacts with the board capacitance $C_{b}$ in this frequency region to produce another resonance. The location of this pole, as suggested by (41), is approximately independent of $C_{d}$. Agreement in this frequency range is still acceptable, although as shown, this final pole may appear at frequencies that are even beyond the second cavity resonance of the unloaded planes.

For still higher frequencies, the full cavity and the LC methods begin to diverge.

\footnotetext{
${ }^{3}$ The denominator of (35) is a second-order system in terms of $\omega$ so the damping of the poles can be obtained from second-order analysis.
}

TABLE II

\begin{tabular}{|c|c|c|c|c|c|}
\hline \multicolumn{3}{|c|}{$\begin{array}{l}\text { Changing No. of Capacitors }(N) \\
\text { with } 100 \text { frequency points }\end{array}$} & \multicolumn{3}{|c|}{$\begin{array}{l}\text { Changing No. of Frequency Points } \\
(F P) \text { with } 20 \text { Capacitors }\end{array}$} \\
\hline$N$ & $\begin{array}{l}\text { Full } \\
\text { Cavity (s) }\end{array}$ & $\begin{array}{l}\text { LC Method } \\
\text { (s) }\end{array}$ & $F P$ & $\begin{array}{l}\text { Full Cavity } \\
\text { (s) }\end{array}$ & $\begin{array}{l}\text { LC } \\
\text { Method (s) }\end{array}$ \\
\hline 50 & 10 & 1 & 200 & 2 & 1 \\
\hline 75 & 15 & 1 & 400 & 4 & 1 \\
\hline 100 & 30 & 1 & 600 & 6 & 1 \\
\hline 125 & 50 & 1 & 800 & 9 & 1 \\
\hline
\end{tabular}

COMPUTATIONAL SPEED COMPARISONS

Execution times (in seconds) for full cavity and LC methods for 100 frequency points (left) and for $N=20$ capacitors (right).

\section{COMPUTATIONAL ASPECTS}

The computation of input and transfer impedances according to $\mathrm{LC}$ method requires the evaluation of (40). This requires an initial eigen mode decomposition of the $\left[L_{N N}\right]$ matrix to obtain the eigen values $\lambda_{i}$ and eigen vectors $v^{i}$. The summations in (36) and are over the $N$ load elements for every frequency point and the algorithm is, therefore, $O[N]$. If there is a change in the placement of only one capacitor, a recalculation of a row or a column in the $\left[L_{N N}\right]$ matrix is required, and thus, a new eigen decomposition is also required. A change in the location of the impedance observation ports $p$ and $q$ does not effect $\left[L_{N N}\right]$, and therefore, no new decomposition needs to be done.

Table II shows the computational advantage gained by using the LC method in contrast with using the full cavity method. The calculations were performed on a $2-\mathrm{GHz}$ Pentium personal computer.

\section{CONCLUSION}

Computationally efficient expressions for the impedance parameters of rectangular power bus structures have been developed. These expressions are valid over a wide frequency range but show specifically the behavior of the supply system at low frequencies. A cylindrical definition of the port has made it possible to identify the low-frequency characteristics of impedance without having to make approximations concerning the geometry of the port, such as it being rectangular or transforming it to a 1-D port. Furthermore, the separation of the low-frequency behavior from an infinite summation of impedance terms has given rise to a fast converging summation that has a quantifiable truncation error and is not influenced by the port size.

The low-frequency components of the impedance expressions were used to develop a method for studying the interactions between loading capacitors and the power ground planes. The low-frequency approach, as expected, gives good results for frequencies below the first cavity mode of the system. However, from the case study presented, it is shown to give good results for frequencies, that are even above the second cavity resonance. This is somewhat surprising as for these frequencies, the unloaded parameters exhibit cavity resonances that are not predicted by the LC impedance. It is possible that the number of capacitors and the regularity of their distribution will affect the upper frequency to which the algorithm can be used. An understanding of the factors would be beneficial. 
As the low-frequency model uses an LC representation for the characteristics of the planes, it only accounts for $1 / \omega$ and $\omega$ frequency trends of the unloaded parameters. This makes it difficult to include copper loss due to skin effect, as this has a $\sqrt{\omega}$ trend. Dielectric losses, however, can be incorporated as a loss tangent in the dielectric of the "C" component of the model.

\section{APPENDIX}

\section{A. Inductance Calculation}

Since

$$
\begin{aligned}
j \sin \left(j k_{n} a\right) & =\frac{-1}{2} \exp \left(\frac{n \pi a}{b}\right)\left(1-\exp \left(\frac{-2 n \pi a}{b}\right)\right) \\
& \approx \frac{-1}{2} \exp \left(\frac{n \pi a}{b}\right)
\end{aligned}
$$

it follows that $L_{2}(x, y)$ of (24) is approximately

$$
\begin{aligned}
L_{2} \approx \frac{\mu d}{\pi} 2 \sum_{n=1}^{\infty}\left[\begin{array}{c}
\cosh k_{n}\left(x_{p}+x-a\right) \\
+\cosh k_{n}\left(\left|x_{p}-x\right|-a\right) \\
n \exp \left(k_{n} a\right)
\end{array}\right] \\
\times \frac{1}{2}\left[\begin{array}{c}
\cos k_{n}\left(y_{p}+y\right) \\
+\cos k_{n}\left(y_{p}-y\right)
\end{array}\right]
\end{aligned}
$$

The total error made in the approximation in (42) is less than $0.2 \%$ (i.e., the largest error is $\exp (-2 \pi)$ for $a=b$ and $N=1$ ).

Since

$$
\begin{aligned}
\frac{\cosh (a) \cos (b)}{\exp (c)} & =\frac{1}{4} \\
& \times\left(\begin{array}{c}
\exp (-a-c-j b)+\exp (-a-c+j b) \\
+\exp (a-c-j b)+\exp (a-c+j b)
\end{array}\right)
\end{aligned}
$$

and $\sum_{n=1}^{\infty} z^{n} / n=-\ln |1-z|$ (see [10]), it is possible to write expressions (25) as (24) with functions $F_{q p}$ and $F_{p p}$ defined as

$$
\begin{aligned}
& F_{q p}\left(x_{p}, y_{p}, x_{q}, y_{q}\right) \\
& =\left(\begin{array}{c}
T_{1}\left(x_{p}+x_{q}, y_{p}+y_{q}\right) \cdot T_{1}\left(x_{p}+x_{q}, y_{p}-y_{q}\right) \cdot \\
T_{1}\left(\left|x_{p}-x_{q}\right|, y_{p}+y_{q}\right) \cdot T_{1}\left(\left|x_{p}-x_{q}\right|, y_{p}-y_{q}\right)
\end{array}\right)
\end{aligned}
$$

$$
F_{p p}\left(x_{p}, y_{p}\right)=T_{2}\left(x_{p}, y_{p}\right) \cdot T_{2}\left(x_{p}, 0\right) \cdot T_{2}\left(0, y_{p}\right) \cdot T_{3}\left(r_{v}\right)
$$

with

$$
\begin{aligned}
T_{1}(x, y)= & \left|1-\exp \left(\pi \frac{x-2 a+j y}{b}\right)\right| \\
& \times\left|1-\exp \left(\pi \frac{-x+j y}{b}\right)\right| \\
T_{2}(x, y)= & \left|1-\exp \left(2 \pi \frac{(x-a)+j y}{b}\right)\right| \\
& \times\left|1-\exp \left(2 \pi \frac{-x+j y}{b}\right)\right| \\
T_{3}\left(r_{v}\right)= & \left|1-\exp \left(\pi \frac{-2 a}{b}\right)\right|\left|\frac{\pi r_{v}}{b}\right| .
\end{aligned}
$$

\section{B. Approximate Error Bound}

An approximate truncation error bound for $M$ terms in the summation on $M_{2}$ in (28) is

$$
|E| \leq \sum_{n=M+1}^{\infty}\left|u\left(x, \sqrt{k^{2}-k_{n}^{2}}\right)-u\left(x, j k_{n}\right)\right| .
$$

Substituting for $u(x, k)$ from (13) and (14) and rearranging gives

$$
|E| \leq \sum_{n=M+1}^{\infty}\left[\begin{array}{c}
\mid U\left(x_{p}+x_{q}-a, \sqrt{k^{2}-k_{n}^{2}}\right) \\
-U\left(x_{p}+x_{q}-a, j k_{n}\right) \mid \\
\mid U\left(\left|x_{p}-x_{q}\right|-a, \sqrt{k^{2}-k_{n}^{2}}\right) \\
-U\left(\left|x_{p}-x_{q}\right|-a, j k_{n}\right) \mid
\end{array}\right] .
$$

Since

$$
\frac{\partial}{\partial x} \frac{\partial}{\partial k} U(k, x)=\frac{(a \operatorname{coth}(a k) \sinh (k x)-x \cosh (k x))}{\sinh (k a)}=0
$$

for $x=a$ and as the second derivative $\partial^{2} / \partial^{2} U(x, k)<0$ for $x=a$, it follows that the maximum error occurs when $x=a$, which is when $x_{q}=x_{p}=a$ in the first term. Since the error in the second term is always less than this, the upper bound is

$$
|E| \leq 2 \sum_{n=M+1}^{\infty} U\left(a, \sqrt{k^{2}-k_{n}^{2}}\right)-U\left(a, j k_{n}\right)
$$

Now

$$
\sqrt{k^{2}-k_{n}^{2}} \approx j k_{n}\left(1-\frac{1}{2}\left(\frac{k}{k_{n}}\right)^{2}\right) .
$$

Let $\Delta_{n}=\frac{1}{2}\left(\frac{k}{k_{n}}\right)^{2}$.

Then

$$
|E| \prec \sum_{n=M+1}^{\infty} \frac{2}{k_{n}}\left[\frac{\cosh \left(a k_{n}\left(1-\Delta_{n}\right)\right)}{\left(1-\Delta_{n}\right) \sinh \left(k_{n} a\left(1-\Delta_{n}\right)\right) a}-\frac{\cosh \left(a k_{n}\right)}{\sinh \left(k_{n} a\right)}\right] .
$$

Since $\cosh \left(k_{n} a\right) \approx 1$ (with error less than $0.37 \%$ for worst case when $a=b$ and $N=0$ ). The $\prec$ sign is used to indicate an approximate bound

$$
\begin{aligned}
|E| & \prec 2 \sum_{n=M+1}^{\infty}\left(\frac{1}{k_{n}\left(1-\Delta_{n}\right)}-\frac{1}{k_{n}}\right) \\
& \succ \frac{k^{2}}{1-\Delta_{N}} \sum_{n=M+1}^{\infty}\left(\frac{1}{k_{n}^{3}}\right) \\
& \prec \frac{k^{2}}{1-\Delta_{M}}\left(\frac{b}{\pi}\right)^{3} \sum_{n=M+1}^{\infty} \frac{1}{n^{3}} \\
& \prec \frac{k^{2}}{1-\Delta_{M}}\left(\frac{b}{\pi}\right)^{3} \int_{M}^{\infty} \frac{1}{n^{3}} d n \\
& \approx \frac{k^{2} b^{3}}{2 M^{2} \pi^{3}}\left(1+\frac{1}{2}\left(\frac{b k}{M \pi}\right)^{2}\right) .
\end{aligned}
$$


Thus, the upper approximate error bound is $O\left[1 / M^{2}\right]$.

\section{Double Summation}

From [1]-[10]

$$
\begin{aligned}
Z_{q p}= & \frac{j \omega \mu d}{a b} \sum_{m=0}^{\infty} \sum_{n=0}^{\infty} \frac{C_{m} C_{n} \operatorname{sinc}^{2}\left(k_{x m} \Delta\right) \operatorname{sinc}^{2}\left(k_{y n} \Delta\right)}{\left(\frac{m \pi}{a}\right)^{2}+\left(\frac{n \pi}{b}\right)^{2}-k^{2}} \\
& \times \cos \left(k_{x m} x_{p}\right) \cos \left(k_{x m} x_{q}\right) \cos \left(k_{y n} y_{p}\right) \cos \left(k_{y n} y_{q}\right)
\end{aligned}
$$

where $k_{x m}=m \pi / a, k_{y n}=n \pi / b$ and $C_{n}=1$ for $n=1$ and $C_{n}=2$ for $n \neq 1$.

\section{REFERENCES}

[1] Y. T. Lo, D. Solomon, and W. F. Richards, "Theory and experiment on microstrip antennas," IEEE Trans. Antennas Propag., vol. AP-27, no. 2, pp. 137-145, Mar. 1979.

[2] G. Lei, R. W. Techentin, and B. Gilbert, "High frequency characterization of power/ground-plane structures," IEEE Trans. Microw. Theory Tech., vol. 47, no. 5, pp. 562-569, May 1999.

[3] G. Lei, R. W. Techentin, P. R. Hayes, D. J. Schwab, and B. Gilbert, "Wave model solution to the power ground plane noise problem," IEEE Trans. Instrum. Meas., vol. 44, no. 2, pp. 300-303, Apr. 1995.

[4] N. Na and M. Swaminathan, "Modeling and transient simulation of planes in electronic packages for $\mathrm{GHz}$ systems," IEEE Trans. Electromagn. Compat., vol. 23, no. 3, pp. 340-352, Aug. 2000.

[5] N. Na and M. Swaminathan, "Modeling and transient simulation of planes in electronic packages for $\mathrm{GHz}$ systems," in Proc. IEEE Conf. Electr. Perform. Electron. Packag., 1999, pp. 149-152.

[6] H. Shim and T. H. Hubing, "A closed-form expression for estimating radiated emissions from the power bus planes in a populated printed circuit board," IEEE Trans. Electromagn. Compat., vol. 48, no. 1, pp. 74-81, Feb. 2006.

[7] Y. Jeong, A. Lu, L. Wei, W. Fan, B. K. Lok, H. Park, and J. Kim, "Hybrid analytic modeling method for split power bus in multilayered package," IEEE Trans. Electromagn. Compat., vol. 48, no. 1, pp. 82-93, Feb. 2006.

[8] L. Wu and C. Tseng, "A theoretical investigation of the resonance damping of magnetic material coating in power/ground plane structures," IEEE Trans. Electromagn. Compat., vol. 47, no. 4, pp. 731-737, Nov. 2005.

[9] R. L. Chen, J. Chen, T. Hubing, and W. Shi, "Analytical model for the rectangular power-ground structure including radiation loss," IEEE Trans. Electromagn. Compat., vol. 47, no. 1, pp. 10-16, Feb. 2005.

[10] M. Hampe and S. Dickmann, "Damping of cavity-mode resonances in PCB power-bus structures using discrete capacitors," IEEE Trans. Electromagn. Compat., vol. 47, no. 4, pp. 880-888, Nov. 2005.

[11] Z. L. Wang, O. Wada, Y. Toyota, and R. Koga, "Convergence acceleration and accuracy improvement in power bus impedance calculation with a fast algorithm using cavity modes," IEEE Trans. Electromagn. Compat., vol. 47, no. 1, pp. 2-9, Feb. 2005.

[12] P. Liu and Z. Li, "An efficient method for calculating bounces in the irregular power/ground plane structure with holes in high-speed PCBs," IEEE Trans. Electromagn. Compat., vol. 47, no. 4, pp. 889-898, Nov. 2005.

[13] J. Trinkle and A. Cantoni, "Impedance analysis of power ground planes loaded with multiple capacitors," in Proc. 16th Int. Zurich Symp. EMC, Zurich, Switzerland, Feb. 2005, pp. 257-259.

[14] J. Trinkle and A. Cantoni, "Efficient impedance calculation of loaded power ground planes," in Proc. 15th Zurich Symp. Electromagn. Compat., Zurich, Switzerland, Feb. 2003, pp. 285-290.

[15] J. Trinkle and A. Cantoni, "Single summation expression for the rectangular power ground plane cavity," in Proc. 16th Int. Zurich Symp. EMC, Zurich, Switzerland, Feb. 2005, pp. 247-250.

[16] J. C. Parker, "Via coupling within parallel rectangular planes," IEEE Trans. Electromagn. Compat., vol. 39, no. 1, pp. 17-23, Feb. 1997.

[17] J. Trinkle, "Analysis of power ground planes" Ph.D. dissertation, Univ. Western Australia, Perth, Australia, Jul. 2005.
[18] V. Mangulis, Handbook of Series for Scientists and Engineers. New York: Academic, 1965.

[19] W. C. Chew, Waves and Fields in Inhomogeneous Media. Piscataway, NJ: IEEE Press, 1990.

[20] T. Okoshi, Planar Circuits for Microwaves and Lightwaves. New York: Springer-Verlag, 1985.

[21] N. W. McLachlan, Bessel Functions for Engineers. Oxford, U.K.: Clarendon, 1955.

[22] C. A. Balanis, Advanced Engineering Electromagnetics. Hoboken, NJ: Wiley, 1989.

[23] P. M. Morse and H. Feshbach, Methods of Theoretical Physics. New York: McGraw-Hill, Part II, 1953.

[24] R. E. Collin, Foundations for Microwave Engineering. New York: McGraw-Hill, 1966.

[25] C. G. Montgomery, R. H. Dicke, and E. M. Purcell, Principles of Microwave Circuits. New York: McGraw-Hill, 1948.

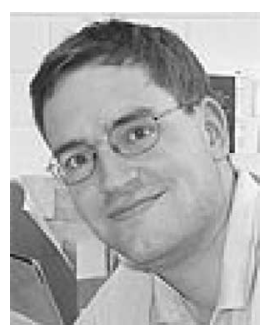

Joe Trinkle was born in Germany on March 8, 1974. He received the B.Sc. degree in mathematics and computer science and the B.E. degree in electrical and electronic engineering from The University of Adelaide, Adelaide, S.A., Australia, in 1994 and 1995, respectively, and the Ph.D. degree in electronic engineering from The University of Western Australia, Perth, W.A., Australia, in April 2006.

From 1996 to 2000, he was with Telstra Research Laboratories, Vic., Australia, where he was engaged in research on electromagnetic compatibility issues relating to asymmetric digital subscriber line. He is currently with the Western Australian Telecommunications Research Center, School of Electrical and Electronic Engineering, The University of Western Australia. His current research interests include power distribution on PCBs and radiation from PCB structures.

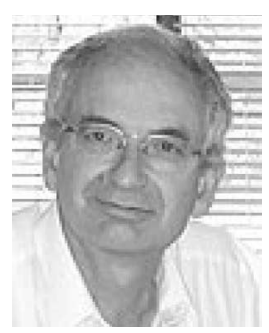

Antonio Cantoni (M'74-SM'83-F'98) was born in Soliera, Italy, on October 30, 1946. He received the B.E. (first class honors) and Ph.D. degrees from The University of Western Australia, Nedlands, W.A., Australia, in 1968 and 1972, respectively.

During 1972, he was a Lecturer of computer science at the Australian National University, Canberra. In 1973, he joined the Department of Electrical and Electronic Engineering, University of Newcastle, Shortland, N.S.W., Australia, where he was the Chair of Computer Engineering until 1986. In 1987, he joined QPSX Communications Ltd., Perth, W.A., as the Director of the Digital and Computer Systems Design Section for the development of the DQDB Metropolitan Area Network. From 1987 to 1990, he was a Visiting Professor in the Department of Electrical and Electronic Engineering, The University of Western Australia, where, since 2000, he has been a Professor of telecommunications, and also the Research Director of the Western Australian Telecommunications Research Center, where, from 1992 to 1997, he was the Director. From 1987 to 1990, he was also a Professor of Telecommunications at the Curtin University of Technology, Perth, W.A., and the Director of the Cooperative Research Centre for Broad-Band Telecommunications and Networking. From 1997 to 2000, he was the Chief Technology Officer of Atmosphere Networks, an optical networks startup that he cofounded. He is currently a Consultant to Sensear Pty Ltd. and Ispire Networks Pty Ltd., startups that he cofounded. His current research interests include adaptive signal processing, electronic system design, phase-locked loops, and networking.

Prof. Cantoni is a Fellow of the Australian Academy of Technological Sciences and Engineering. He has also been an Associate Editor of the IEEE TRANSACTIONS ON SIGNAL PROCESSING. 